\title{
Editorial Acknowledgement
}

The Behavioral and Brain Sciences and Cambridge University Press are very pleased to acknowledge a grant from the Alfred P. Sloan Foundation. This award will defray some of the extraordinary editorial costs involved in the establishment and implementation of the BBS project. It will also contribute to strengthening and expanding the BBS open peer commentary service through the use of computer-assisted bibliographic retrieval methods for selecting topics, authors and commentators.

We are grateful to the Foundation for its generous support at this critical stage in the development of the BBS treatment process to enhance communication in the cognitive sciences. 


\section{BBS Associateship}

Qualified professionals in the biobehavioral sciences who have either (1) been nominated by a current BBS Associate, (2) refereed for BBS, or (3) had a commentary or article accepted for publication are eligible to become BBS Associates. Editors of learned journals and officers of learned societies in the biobehavioral sciences are invited to become BBS Associates ex officio. Detailed protocol cards indicating each Associate's specialization and interest are maintained by BBS to aid in commentator selection. Associates have a direct role in shaping BBS policy and, starting with the 1980 volume, are eligible to receive the journal at a special rate. Please write to the editor for further information. BBS Associates as of October 1979 .

Abler, Wm. L. (Humanities) Ill. Inst. Tech. Adolph, Alan R. (Neurosci.) Eye Res. Inst. Albert, D.J. (Psychology) Univ. Brit. Columbia Ainsworth, Mary D. Salter (Psychology) Univ. Virginia Altmann, Stuart (Biology) Univ. Chicago

Anderson, Robert M. Jr. (Philosophy) Rensselaer Poly. Inst Anderson, Samuel W. (Comm. Sci.) New York St. Psych. Inst. Andreae, J.H. (Electr. Engineering) Univ. Christchurch Andrew, R.J. (Biology) Univ. Sussex

Applewhite, Philip B. (Biology) Yale Univ.

Arbib, Michael A. (Comp. \& Info. Sci.) Univ. Mass. Atherton, Margaret (Philosophy) Univ. Maryland Austin, George (Neurosurgery) Loma Linda Univ. Sch. Med. Avila, Raul (Linguistics) Colegio Mexico Babad, Elisha (Education) Hebrew Univ. Jerusalem Bakan, David (Psychology) York Univ. Bakan, Paul (Psychology) Simon Fraser Univ. Bakker, Dirk J. (Neuropsychology) Paedologisch Inst. Balaban, P. (Neuro. Inst.) U.S.S.R. Acad. Sci. Baldwin, Janice 1. (Sociology) Univ. Calif Baldwin, John D. (Sociology) Univ. Calif Balonov, L.). (Sechenov Inst.) U.S.S.R. Acad. Sci. Barber, T.X. (Psychology) Cushing Hosp. Barkow, Jerome H. (Sociology) Dalhousie Univ. Barlow, George W. (Zoology) Univ. Calif. Baron, Robt. J. (Math.) Univ. lowa

Bart, William M. (Education) Univ. Minn. Bates, Elizabeth (Psychology) Univ. Colorado Baxter, Claude F. (Neurochemistru) Sepulveda V.A. Hosp. Beaumont, J.G. (Psychology) Univ. Leicester Beck, Benjamin B. Brookfield Zoo

Beloff, John (Psychology) Univ. Edinburgh Belshaw, Cyril S. (Anthropology) Univ. Brit. Columbia ex off. Belth, Marc (Psychology) C.U.N.Y.

Ben-Ari, Y. (Neurophysiology) C.N.R.S.

Bennett, M.V.L. (Neuroscience) Einstein Coll. Med.

Berlucchi, Giovanni (Physiol.) Univ. Pisa

Berndt, Thomas J. (Psychology) Yale Univ.

Bernstein, Irwin S. (Psychology) Univ. Georgia

Bertelson, P. (Psychology Exp.) Univ. Libre Bruxelles Bever, Thomas G. (Psychology) Columbia Univ. Bibikov, Nicolaj (Acoustics) U.S.S.R. Acad. Sciences Bierwisch, Manfred (DDR) Akad. Wissenschaften Bigelow, Julian Inst. Advanced Study Bindra, Dalbir (Psychology) McGill Uniy. Bishop, P.O. (Physiology) Austral. Nat. Univ. Bitterman, M.E. (Sens. Sci.) Univ. Hawaii Bizzi, Emilio (Psychology) Mass. Inst. Tech. Blakemore, Colin (Physiology) Cambridge Univ. Blanchard, C. (Psychology) Univ. Hawaii

Blanchard, R. (Psychology) Univ. Hawai Blank, Marion (Psychiatry) Rutgers Med. Sch. Block, Ned (Philosophy) Mass Inst. Tech. Bloom, Lois (Psychology) Columbia Univ. Blough, Donald S. (Psychology) Brown Univ.
Blumenthal, Arthur L. (Psychology) Univ. Mass. Boden, Margaret A. (Cognitive Studies) Univ. Sussex Bogen, Joseph E. Ross Loos Medical Group Boller, Francois (Neurolog $y$ ) Pittsburgh V.A. Hosp Bolles, Robert C. (Psychology) Univ. Washington Booth, D.A. (Psychology) Univ, Birmingham Borbely, A.A. (Pharmacol.) Univ. Zurich Borgia, Gerald (Biology) Univ. Chicago Bornstein, Marc H. (Psychology) Princeton Univ. Bowsher, David (Neurobiology) Univ. Liverpool Boynton, Robert (Psychology) Univ. Calif. San Diego Bradshaw, John L. (Psychology) Monash Univ Brain, P.F. (Zoology) Univ. Coll. Swansea Brainerd, Charles J. (Psychology) Univ. Western Ontario Bremner, Gavin (Psychology) Univ. Lancaster Bridgeman, Bruce (Psychobiology) Univ. Calif. Santa Cruz Bromberger, Sylvain (Philosophy) Mass. Inst. Tech

Brown, Jerram L. (Biology) S.U.N.Y. Brown, Robert T. (Psychology) Univ. North Carolina Brown, Roger (Psychology) Harvard Univ. Bryden, M.P. (Psychology) Univ. Waterloo Bubenik, A.B. (Behavioral Biology) Ont. Min. Nat. Res. Bunge, Mario (Philosophy) McGill Univ. Burdsal, Charles (Psychology) Wichita State Univ. ex off. Bures, Jan (Physiology) Czechoslovak Acad. Sci. Burghardt, Gordon M. (Psychology) Univ. Tennessee Burke, R.E. (Neurophysiology) N.I.H. Buser, P. (Neurophysiology) Univ. Curie Buss, Allan R. (Psychology) Univ. Calgary Butter, C.M. (Neuroscience) Univ. Mich. Cairns, Robert B. (Psychology) Univ. No. Carolina Caldwell, Roy L. (Zoology) Univ. Calif. Campbell, Donald (Psychology) Syracuse Univ. Campbell, Bernard, Norfolk Campbell, J.A. (Computer Sci.) Exeter Univ Candland, Douglas K. (Psychology) Bucknell Univ. Caplan, David (Neuropsychology) Ottawa Capranica, Robert R. (Neurobiology) Cornell Univ. Carey, Susan (Psychology) Mass. Inst. Tech Carlier, Michele (Psychology) Univ. Paris Carlton, Peter (Psychiatry) Rutgers Med. Sch Catania, A. Charles (Psychology) Univ. Md. Balt. Cty. Cernáček, J. (Neurology) Comenius Univ. Med. Sch. Cervetto, Luigi (Neurophysiology) C.NR

Chalmers, N.R. (Biology) Open Univ Chapman, Loring F. (Behavioral Biology) Univ. Calif. Med. Sch. Chapple, William D. (Biological Sci.) Univ. Connecticut Charlesworth, William (Child Development) Univ. Minnesota Charniak, Eugene (Computer Science) Brown Univ. Chaurasia, B.D. (Anatomy) Gajra Raja Medical Coll. Chiarelli, B., Inst. Anthro. Torino ex off. Chomsky, Noam (Linguistics) Mass. Inst. Tech. Chorover, Stephan L. (Psychology) Mass. Inst. Tech Chow, Kao Liang (Neurology) Stanford Univ. Med. Ctr. Chubin, Daryl E. (Social Science) Georgia Inst. Tech. Churchland, P.M. (Philosophy) Univ. Manitoba Churchland, P.S. (Philosophy) Univ. Manitoba Chute, Douglas L. (Psychology) Univ. Otago Codeceira, Alcides, Jr., Ed., Neurobiologia, ex. off. Cohen, L. Jonathan (Philosophy) Queen's Coll., Oxford Univ. Cole, Ronald A. (Psychology) Carnegie-Mellon Univ. Collins, John K. (Psychology) Macquarie Univ. Collins, Robert L. (Behav. Genetics) Jackson Lab. Coltheart, Max (Psychology) Birbeck, Coll Cooper, William E. (Psychology) Mass. Inst. Tech Corazza, R. (Physiol.) Univ. Ferrara Corballis, Michael C. (Psychology) Univ. Auckland 
Coren, Stanley (Psychology) Univ. Brit. Columbia Cornell, Edward H. (Psychology) Univ. Alberta Corner, M.A., Netherlands Cent. Inst. Brain Res Coscina, Donald V. (Biopsychology) Clarke Inst. Psychiatry Cosyns, M., Licq, France

Covian, Miguel R. (Physiology) Univ. São Paulo Med. Sch. Craske, Brian (Psychology) Mem. Univ. Newfoundland Creutzfeldt, O. (Neurobiology) Max-Planck-Institut Cromer, Richard (Psychology) Med Res. Council Crow, T.J. (Psychiatry) Clinical Res. Cent Cushing, Steven, Higher Order Software Inc Daitzman, Reid J., Stamford

Damasio, Antonio R. (Neurology) Univ. Iowa Hosp.

Danks, Joseph H. (Psychology) Kent State Univ. Davidson, Donald (Philosophy) Univ. Chicago Davidson, Richard J. (Psychology) S.U.N.Y Davis, John D. (Psychology) Univ. Illinois Davis, Lawrence H. (Philosophy) Univ. Missouri Davis, William J. (Thimann Labs.) Univ. Calif Dawkins, Marian (Zoology) Univ. Oxford Day, R.H. (Psychology) Monash Univ

Deglin, V.L. (Sechenov Inst.) U.S.S.R. Acad. Sci

Deich, Ruth F., Inst. Res. Human Growth

Del Cerro, M. (Brain Res. Ctr.) Univ. Rochester Delgado, Jose M.R. (Neuroscience) Ctr. Ramon Y Cajal Dennett, D.C. (Philosophy) Tufts Univ.

Desmedt, John E. (Brain Res.) Univ. Brussels, ex off. Dev, Parvati (Rehab. Engr.) Palo Alto V.A. Hosp Diamond, I.T. (Psychology) Duke Univ.

Diamond, Marian C. (Physiology-Anatomy) Univ. Calif. Dienske, Herman, Rijswijk Primate Ctr.

Dimond, Stuart J. (Psychology) Univ. Coll. Cardiff Dingwall, William Orr (Linguistics) Univ. Maryland Dismukes, R. Key (Vision Comm.) Natl. Res. Council

Dolhinow, P. (Anthropology) Univ. Calif.

Domotor, Zoltan (Philosophy) Univ. Pennsylvania Donchin, Emanuel (Psychology) Univ. Illinois

Doty, Robert W. (Brain Res. Ctr.) Univ. Rochester Douglas, Robert J. (Psychology) Univ. Washington Drachman, G. (Linguistics) Univ. Salzburg Dreyfus, Hubert L. (Philosophy) Univ. Calif. Duysens, J., AALST

Dykman, R.A. (Beh. Sci.) Univ. Arkansas

Eaves, Lindon (Psychology) Univ. Oxford

Ebenholtz, Sheldon M. (Psychology) Univ. Wisconsin

Eccles, John C., Cá A La Grá

Economos, Judith, Renaissance Studio, Scarsdale

Eibl-Eibesfeldt, I. (Human Ethology) Max-Planck-Institut

Eichelman, Burr, Middleton Memorial V.A. Hosp.

Eidelberg, E. (Surgery) Univ. Texas Health Sci. Ctr.

Eisenberg, John F., National Zoological Park

Eiserer, Leonard A. (Psychology) Franklin \& Marshall Coll

Ellis, Hadyn (Psychology) Univ. Aberdeen

Ellsworth, Phoebe C. (Psychology) Yale Univ.

Ennis, Robert H. (Education) Univ. Illinois

Epstein, Herman T. (Biology) Brandeis Univ.

Epstein, R. (Psychology) Harvard Univ.

Ettlinger, G., Inst. Psychiatry

Evans, Jonathan (Behav. Social Science) Plymouth Poly.

Evoy, W.H. (Biology) Univ. Miami

Eysenck, Hans I. (Psychology) Inst. Psychiatry, London

Farrell, B.A. (Corpus Christi Coll.) Univ. Oxford

Favazza, A.R. (Psychiatry) Univ. Missouri Med. Sch.

Fentress, John C. (Psychology) Dalhousie Univ.

Feth, L.L. (Audiology) Purdue Univ.

Fillmore, C. (Linguistics) Univ. Calif

Finke, Ronald A. (Psychology) Cornell Univ.

Finley, Gordon E. (Psychology) Florida International Univ., ex off.

Fischer, Kurt W. (Psychology) Univ. Denver

Fishbein, William (Psychology) C.U.N.Y.

Fiske, Donald W. (Beh. Sci.) Univ. Chicago

Fitzgerald, H.E. (Psychology) Michigan State Univ.

Fitzsimons, J.T. (Physiological Lab.) Univ. Cambridge

Flavell, John H. (Psychology) Stanford Univ.

Fletcher, Paul (Linguistics) Univ. Reading

Flores D'Arcais, G.B. (Psychology) Univ. Leiden

Fodor, J.A. (Psychology) Mass. Inst. Tech.

Fonberg, Elzbieta (Neurophysiology) Nencki Inst.
Fouts, Roger S. (Psychology) Oklahoma Univ. Fraisse, P. (Psychology) Univ. Rene Descartes Fraser, Peter J. (Zoology) Univ. Aberdeen Freemon, Frank R. (Neurology) Nashville V.A. Hosp. Freides, David ( $P$ sycholog $y$ ) Emory Univ. Freund, H.J. (Neurology) Univ. Dusseldorf Friedhoff, Arnold J. (Millhauser Labs.) N.Y. Univ. Med. Ctr. Frigyesi, T.L. (Physiology) Texas Tech. Univ. Med. Sch. Frijda, Nico H. (Psychology) Univ. Amsterdam Frumkes, Thomas E. (Psychology) C.U.N.Y Fukuda, Y. (Neurophysiology) Osaka Univ. Med. Sch. Fuller, John L., York Furedy, John J. (Psychology) Univ. Toronto Gadlin, Howard (Psychology) Univ. Mass. Gaines, B.R. (Elect. Eng. Sci.) Univ. of Essex Galbraith, Gary (Neuropsychology) Pacific St. Hosp. Gandelman, Ronald (Psychology) Rutgers Univ. Gardner, Howard (Education) Harvard Univ. Gazzaniga, M.S. (Neurology) Cornell Med. Ctr. Gedye, John L. (Neurology) Wayne St. Univ. Geffen, Gina (Psychology) Flinders Univ. So. Australia Geist, V. (Envir. Des.) Univ. Calgary Ghiselin, Michael T., Univ. Calif. Bodega Mar. Lab. Gibson, Kathleen R. (Anatomy) Univ. Texas

Gilbert, R.M. (Psychology) Addiction Res. Foundation Glantz, Raymon (Biology) Rice Univ.

Glasersfeld, E.V. (Psychology) Univ. Georgia Glass, Arnold L. (Psychology) Rutgers College Glass, Gene V. (Education) Univ. Colorado

Glenn, Loyd L. (Psychiatry) Stanford Univ. Med. Sch Glickstein, Mitchell (Psychology) Brown Univ. Globus, Gordon G. (Psychiairy) Univ. Calif. Glucksberg, S. (Psychology) Princeton Univ Goguen, J.A. (Computer Science) Univ. Calif Goldstein, Leonide (Psychiatry) Rutgers Med. Sch Goodglass, Harold, Boston V.A. Hosp. Goodluck, Helen (English) Univ. Wisc Goodwin, B.C. (Biology) Univ. Sussex Gottlieb, Gilbert (Psychology) Dorothea Dix Hosp. Gould, James L. (Biology) Princeton Univ. Gould, S.J. (Agassiz Museum) Harvard Univ. Gourevitch, George (Psychology) Hunter Coll. Granit, Ragnar (Neurophysiology) Karolinska Inst. Grastyán, Endre (Physiology) PECS Univ. Med. Sch Gray, I.A. (Psychologu) Univ. Oxford

Green, Michael B. (Philosophy) Univ. Texas Greenberg, Gary (Psychology) Wichita St. Univ. Greenberg, Ramon (Psychiatry) Boston Univ. Hosp. Greenblatt, S.H. (Neuroscience) Medical Coll. Ohio Greenfield, Patricia (Psychology) Univ. Calif. Greenwald, A.G.-Editor (Psychology) Ohio St. Univ., ex off. Grene, Marjorie (Philosophy) Univ. Calif. Davis Griffin, Donald, Rockefeller Univ.

Grigg, Peter (Physiology) Univ. Mass. Med. Ctr. Grillner, Sten (Physiology) Karolinska Inst. Gross, Charles G. (Psychology) Princeton Univ. Grossberg, Stephen (Mathematics) Boston Univ. Groves, Colin P. (Anthropology) Australian Natl. Univ. Gruber, Howard E. (Cognitive Studies) Rutgers Univ. Grüsser, O.J. (Physiology) Free Univ. Berlin Guerrier, P. (Biology) French Min. Natl. Ed. Guilleminault, C. (Sleep Disorders Center) Stanford Univ. Gunnar, Megan (Beh. Sci.) Stanford Univ. Sch. Med Guth, S.L. (Psychology) Indiana Univ. Gyr, John W. (Psychiatry) Univ. Michigan Haber, Ralph Norman (Psychology) Univ. Illinois Hailman, Jack P. (Zoology) Univ. Wisconsin Hall, W.G. (Psychobiology) Dorothea Dix Hosp. Halwes, T., Haskins Labs.

Handy, Rollo, Behavioral Res. Council Haralick, Robert (Computer Sci.) Univ. Kansas Hardyck, Curtis (Education) Univ. Calif. Harman, Gilbert (Philosophy) Princeton Univ Harmon, Leon (Biomed. Engr.) Case Western Reserve Univ. Harrington, Gordon M. (Psychology) Univ. Northern Iowa Harris, L..J. (Psychology) Michigan State Univ. Harvey, Lewis O. Jr. (Psychology) Univ. Colorado Hastie, Reid (Psychology) Harvard Univ. 
Hatton, Glenn L. (Psych. \& Zoology) Michigan State Univ Haugeland, John (Philosophy) Univ. Pittsburgh Hausfater, Glenn (Liddell Lab.) Cornell Univ.

Hayes, P.J. (Computer Sci.) Univ. Essex Hebb, D.O. (Psychology) Dalhousie Univ. Heffner, John (Philosophy) Lebanon Valley College Hendersen, Robert W. (Psychology) Univ. Illinois Henle, Mary, New Sch. Social Res.

Henn, Volker (Neurological Inst.) Univ. Zurich Henriksen, S.J., Salk Inst.

Herberg, L.J. (Neurolog $y$ ) National Hosp.

Hermann, A. (Biology) Konstanz Univ.

Hicks, R.E., Univ. Lethbridge

Hilgard, E.R. (Psychology) Stanford Univ.

Hill, David R. (Comp. Sci.) Univ. Calgary

Hines, Terence (Neurology) Cornell Med. Coll.

Hippler, Arthur E., Ed. Journal Psychological Anthropology, ex off.

Hirano, Tetsuya (Ocean Res. Inst.) Univ. Tokyo

Hirsch, Helmut V.B. (Biology) S.U.N.Y

Hirsch, Jerry (Psychology) Univ. Illinois

Hoebel, Bartley (Psychology) Princeton Univ.

Holloway, Ralph L. (Anthropology) Columbia Univ.

Hooker, C.A. (Philosophy) Univ. Western Ontario

Hooper, Frank H. (Sch. Family Res.) Univ. Wisconsin

Hopkins, Brian (Neurology) Groningen Univ. Hosp.

Horn, Berthold D.P. (Artif. Intell. Lab.) Mass. Inst. Tech.

Horrell, R.I. (Psychology) Univ. Hull

Horridge, G.A. (Bio. Sci.) Australian Natl. Univ.

Hoyle, Graham (Biology) Univ. Oregon

Hsiao, Sigmund (Psychology) Univ. Arizona

Hubbard, John I. (Physiology) Univ. Otago

Hubel, David H. (Neurobiology) Harvard Med. Sch.

Huber, Franz, Max-Planck-Inst

Hudson, Patrick T.W. (Psychology) Nijmegen Univ.

Hull, David L. (Philosophy) Univ. Wisc.

Hulse, Stewart H. (Psychology) Johns Hopkins Univ.

Hunsperger, R. (Physiology) Univ. Zurich

Iles, J.F. (Physiology) Oxford Univ.

Immelmann, Klaus (Ethology) Univ. Bielefeld

Ingle, David (Neurophysiology) McLean Hosp.

Ingvar, D.H. (Neurophysiology) Univ. Lund Hosp.

Irwin, Francis W. (Psychology) Univ. Penn.

Isaacson, Robert L. (Psychology) S.U.N.Y.

Isard, S. (Psychology) Sussex Univ.

Ivanov, V.V., (Typology) USSR Acad. Sci

Iwama, K. (Neurophys.) Osaka Univ. Med. Sch.

Izard, Carroll E. (Psuchology) Univ. Delaware

Jacobson, Marcus (Anatomy) Univ. Utah Med. Ctr.

Jaynes, Julian (Psychology) Princeton Univ.

Jeannerod, Marc (Neuropsychology) I.N.S.E.R.M.

Jeffrey, W.E. (Psychology) Univ. Calif. Los Angeles

Jenkins, James J. (Human Learn. Res. Ctr.) Univ. Minn.

Jerison, Harry J. (Psychiatry) Univ. Calif.

Johnson, A.K. (Psychology) Univ. Iowa

Johnson, Richard F.Q. (Psychology) U.S. Army Natick Res. \& Dev.

Johnson, Ronald W. (Psychology) St. Francis Xavier Univ.

Johnson-Laird, P. (Psychology) Sussex Univ.

Jones, Barbara E. (Neuroscience) Montreal Neurological Hosp.

Jones, Edward E. (Psychology) Princeton Univ.

Jones, E.G. (Anatomy) Washington Univ. Sch. Med.

Joshi, Aravind K. (Computer Sci.) Univ. Penn.

Jouvet, M. (Exp. Medicine) Univ. Claude Bernard

Julesz, B., Bell Laboratories

Kaas, Jon H. (Psychology) Vanderbilt Univ.

Kahneman, Daniel (Psychology) Univ. Brit. Columbia

Kaiser, Peter K. (Psychology) York Univ.

Kalverboer, Alex (Psychology) Groningen Univ. Hosp.

Karmiloff-Smith, A. (Psychology) Univ, Geneva

Kasinov, Vadim B. (Lenigrad Nuc. Phys. Inst.) U.S.S.R. Acad. Sci.

Kater, Stanley B. (Zoology) Univ. Iowa

Katz, Jerrold J. (Linguistics) C.U.N.Y.

Kaufman, Lloyd (Psychology) New York Univ.

Kaufman, I. Charles (Psychiatry) Univ. Colorado Med. Ctr

Kelly, John S. (Pharmacology) Cambridge Med. Sch.

Kelso, J.A. Scott, Haskins Lab.

Kempen, Gerard (Psychology) Katholieke Univ., Nijmegen

Kendler, Howard H. (Psychology) Univ. Calif.

Kendler, Tracy S. (Psychology) Univ. Calif.
Kenshalo, Dan R. (Psychology) Florida St. Univ.

Kessen, William (Psychology) Yale Univ.

Killeen, P.R. (Psychology) Arizona St. Univ.

Kimmel, H.D. (Psychology) Univ. South Florida

Kinsbourne, Marcel, Hosp. Sick Children

Klahr, David (Psychology) Carnegie-Mellon Univ.

Klima, Edward S. (Linguistics) Univ. Calif. San Diego

Klingsporn, M.J. (Psychology) Wichita St. Univ.

Klitzner, Michael (Psychology) Univ. Wisc.

Klopfer, Peter H. (Zoology) Duke Univ.

Kohler, Ivo (Psychology) Univ. Innsbruck

Kohn, Herb (Psychiatry) Rutgers Med. Sch.

Kohout, Ladislav J., Colchester

Kolers, Paul A. (Psychology) Univ. Toronto

Kolk, Herman H.J. (Psychology) Katholieke Univ. Nijmegen

Kopp, N. (Neuropathology) Hosp. Neurologique

Korenovsky, Anna (Neuroscience) New York St. Psychiatric Inst.

Koukou-Lehmann, M. (Psychiatry) Univ. Zurich

Kovach, Joseph K., Menninger Foundation

Krasner, Leonard (Psychology) S.U.N.Y.

Krauthamer, George (Anatomy) Rutgers Med. Sch

Krippner, Stanley, Humanistic Psychology Inst.

Kruger, Lawrence (Anatomy) Univ. Calif.

Kruglanski, Arie W. (Psychology) Tel-Aviv Univ.

Kuhn, Thomas S. (Philosophy) Princeton Univ.

Kummer, Hans (Zool. Inst.) Univ. Zurich

Kupfermann, Irving (Physiology) Columbia Col. Phys. Surg.

Kurtines, William M. (Psychology) Florida Int. Univ.

Kutscher, Charles L. (Psychology) Syracuse Univ

Kuypers, H.G.J.M. (Anatomy) Erasmus Univ

Laborit, H. (Lab. D'Eutonologie) Hop. Boucicaut

Laferriere, D., Ed. Semiotic Scene, ex off.

Lakoff, George (Linguistics) Univ. Calif.

Landes, Susan (Anthropology) Univ. Calif.

Lappin, J.S. (Psychology) Vanderbilt Univ.

Larimer, J.L. (Zoology) Univ. Texas

Lawton, J.T. (Sch. Family Res.) Univ. Wisconsin

Lea, S.E.G. (Psychology) Univ. Exeter

Lecours, André Roch, Hôtel-Dieu Montréal

Leibovic, K.N., Neurosci. Res. Prog., Mass. Inst. Tech.

Lemay, Marjorie (Radiology) Harvard Med. Sch.

Lenat, D.B. (Computer Sci.) Stanford Univ.

Lenherr, Fred K., Berkeley Brain Center

Lerner, Richard M. (Ctr. Adv. Study Beh. Sci.) Stanford Univ

Levelt, William J.M. (Psycholing) Max-Planck-Gesellschaft

Levis, Donald J. (Psychology) S. U.N.Y.

Levitt, M. (Physiology) Bowman Gray Sch. Med.

Lévy-Schoen, Ariane (Psychology) Univ. Curie

Lewis, E.R. (Computer Sci.) Univ. Calif.

Lewis, Michael (Except. Child.) Educational Testing Serv

Liberman, Alvin M., Haskins Lab.

Libet, Ben (Physiology) Univ. Calif.

Limber, John (Psychology) Univ. New Hampshire

Linde, Charlotte, Structural Semantics Inc.

Lipp, H.P. (Anatomy) Univ. of Lausanne

Lipsitt, Lewis P. (Psychology) Brown Univ

Lissák, K., Pecs Med. Univ.

Llinás, R. (Physiology) New York Univ. Med. Ctr.

Lockard, Joan S. (Neuro. Surg.) Univ. Wash.

Loeb, G. (Public Health) N.I.H

Longuet-Higgins, C. (Psychology) Sussex Univ.

Luco, J.V. (Neurophysiology) Univ. Catolica Chile

Ludlow, A.R. (Zoology) Imperial Coll.

Lynch, James C. (Physiology) Mayo Grad. Sch. Med.

Lyons, W.E. (Philosophy) Univ. Glasgow

Mack, Arien (Psychology) New Sch. Soc. Res.

Mackintosh, N.J. (Psychology) Sussex Univ.

MacWhinney, Brian (Psychology) Univ. Denver

Madsen, K.B., Copenhagen

Magalhães-Castro, B. (Animal Biology) Univ. Brazil

Main, Mary (Psychology) Univ. of Calif

Malatesha, R.N. (Education) Oregon St. Univ.

Mandler, George (Psychology) Univ. Calif.

Mansfield, Richard J.W. (Psychology) Harvard Univ.

Marg, Elwin (Optometry) Univ. Calif.

Marino, Matthew (English) Univ. Alabama

Markl, Hubert (Biology) Univ. Konstanz

Markman, Ellen M. (Psychology) Stanford Univ. 
Marks, Lawrence E., Pierce Found. Lab. Marler, Peter, Rockefeller Univ.

Marr, D. (Artif. Intell.) Mass. Inst. Tech.

Marriott, F.H.C. (Biomathematics) Oxford Univ

Marshack, A. (Peabody Museum) Harvard Univ

Marshall, John C. (Psycholing) Univ. Nijmegen

Massaro, D.W. (Psychology) Univ. Wisc.

Masters, John C. (Child Dev.) Univ. Minn.

Matin, Leonard (Psychology) Columbia Univ.

Matthews, R.J. (Philosophy) Cook Coll.

Maxwell, Grover (Philos. Sci.) Univ. Minn

Mayo, Robert J. (Psychology) Ball St. Univ.

Mazur, James E. (Psychology) Yale Univ.

McCarley, Robert W. (Neurophys.) Mass. Mental Hlth. Ctr.

McCarthy, John (Artif. Intell. Lab.) Stanford Univ.

McCawley, James (Linguistics) Univ. Chicago

McCloskey, D.I. (Physiology) Univ. New South Wales

McClure, William O. (Biology) Univ. Southern Calif.

McFadden, Dennis (Psychology) Univ. Texas

McNeill, David (Beh. Sci.) Univ. Chicago

Meerson, J.A. (Sechenov Inst.) U.S.S.R. Acad. Sci.

Meier, Gilbert W., Ed. Developmental Psychobiology, ex off

Mellgren, Roger L. (Psychology) Univ. Oklahoma

Melzack, Ronald (Psychology) McGill Univ.

Menzel, Emil W. (Psychology) S.U.N.Y

Metz, John T. (Biology) Ill. State Psych. Inst

Meułders, Michel (Neurophysiol.) Univ. Catholique Louvain

Miczek, Klaus A. (Psychology) Carnegie-Mellon Univ.

Mikaelian, H.H. (Psychology) Univ. New Brunswick

Milgram, N.W. (Psychology) Scarborough Coll.

Mill, Peter J. (Zoology) Univ. Leeds

Miller, George A. (Psychology) Princeton Univ.

Miller, Joel M. (Psychology) Johns Hopkins Hosp.

Mineka, Susan (Psychology) Univ. Wisc.

Miner, Earl (English) Princeton Univ.

Mishkin, Mortimer (Neuropsychol.) N.I.M.H.

Mittwoch, Ursula (Human Genetics) Univ. Coll. London

Molfese, Dennis L. (Psychology) Southern Illinois Univ.

Morgan, Michael J. (Psychological Lab.) Univ. Cambridge

Morgane, Peter J., Worcester Fdn. Exp. Bio.

Morrell, Roger M. (Neurology) Allen Park V.A. Hosp.

Morrison, Adrian (Veterinary Med.) Univ. Penn.

Morton, Adam (Philosophy) Univ. Bristol

Morton, John (Psychology) Med. Res. Council

Moshman, David (Educ. Psych.) Univ. Nebraska

Mpitsos, George (Anatomy) Case Western Reserve Univ. Sch. Med.

Murray, Edward J. (Psychology) Univ. Miami

Murthy, K.S. Krishna, Texas Med. Center

Nakajima, Shinshu (Psychology) Univ. Tokyo

Naquet, R. (Physiology) Georgian C.N.R.S.

Narikashvili, S.P. (Physiology) Acad. of Sci

Natani, Kirmach (Neuropsychol.) U.S.A.F. Sch. Aerospace Med.

Natsoulas, Thomas (Psychology) Univ. Calif.

Navarick, D. (Psychology) Calif. St. Univ.

Negrao, Nubio (Neurophysiol.) Univ. Sao Paulo

Neimark, Edith D. (Mnemonics Res. Ctr.) Douglass Coll

Nelson, Katherine (Develop. Psychol.) C.U.N.Y.

Nelson, Keith E. (Psychology) Penn. St. Univ.

Ness, A.R. (Physio.) Univ. Coll. London

Neville, A.C. (Zoology) Univ. Bristol

Nïcolaidis, Stylianos (Neurobiology) C.N.R.S

Nicolayenko, N.N. (Sechenoo Inst.) U.S.S.R. Acad. Sci

Nilsson, Nils J. (Artif. Intell. Ctr.) Stanford Res. Inst.

Nishihara, H.K. (Artif. Intell. Lab.) Mass. Inst. Tech.

Noback, Charles (Anatomy) Columbia Coll. Phys. Surg.

Nonneman, Arthur J. (Psychology) Univ. Kentucky

Norman, Donald A. (Psychology) Univ. Calif.

Nosanchuk, T.A. (Sociology) Carleton Univ.

Notterman, Joseph (Psychology) Princeton Univ.

Ochs, Elinor (Linguistics) Univ. So. Calif

Öhman, A. (Psychology) Univ. Bergen

Öhman, Sven (Linguistics) Uppsala Univ

O'Kelly, Lawrence I. (Psychology) Michigan St. Univ.

Olson, David, Ont. Inst. Studies Educ.

Oniani, T. (Physiology) Georgian Acad. Sci

Orne, Martin T. (Exp. Psychiatry) Penn. Hosp.

Ortony, A. (Reading Ctr.) Univ. Ill

Osherson, Daniel N. (Psychology) Mass. Inst. Tech.
Oswaldo-Cruz, E. (Neurobio.) Univ. Rio de Janeiro Otto, Herbert R. (Philosophy) Plymouth St. Coll.

Overstreet, David H. (Biol. Sci.) Flinders Univ. So. Australia

Ozer, Mark N. (Child. Devel.) Geo. Washington Sch. Med.

Paap, K.R. (Psychology) New Mex. St. Univ.

Page, Stewart (Psychology) Brockville Psychiatric Hosp

Paillard, J. (Psychophys.) C.N.R.S.

Panksepp, Jaak (Psychology) Bowling Green St. Univ.

Parisi, Domenico (Psychology) C.N.R.

Parker, Sue Taylor (Anthropology) Sonoma St. Coll

Pascual-Leone, Juan (Psychology) York Univ.

Pasik, Pedro (Neurology) Mt. Sinai Sch. Med.

Pasik, Tauba (Neurology) Mt. Sinai Sch. Med.

Pask, Gordon, System Research Ltd.

Passman, Richard H. (Psychology) Univ. of Wise.

Pearlman, Chester A., Brookline V.A. Hosp.

Peck, Jeffrey W. (Psychology) Univ. Utah

Perry, John R. (Philosophy) Stanford Univ.

Perzigian, Anthony J. (Anthropology) Univ. Cincinnati

Peterson, Steven A. (Political Science) Alfred Univ.

Petrinovich, Lewis (Psychology) Univ. Calif

Picton, T.W. (Medicine) Univ. Ottawa

Pinard, Adrien (Psychology) Univ. Montreal

Pinkava, V. (Psychology) Severalls Hosp.

Pinker, Steven (Cog. Sci. Ctr.) Mass. Inst. Tech.

Poggio, T. (Neurobiol.) Max-Planck-Inst

Pollack, Robert H. (Psychology) Univ. Georgia

Porac, Clare (Psychology) Univ. Victoria

Powers, William T., Northbrook

Preilowski, Bruno (Psychology) Univ. Konstanz

Premack, David (Psychology) Univ. Penn.

Prescott, James W. (Dev. Psychobiol.) N.I.C.H.H.D

Pribram, Karl (Psychology) Stanford Univ.

Prochazka, A. (Physiology) St. Thomas Hosp. Med. Sch.

Pubols, L.M. (Anatomy) Penn. Med. Coll.

Puccetti, Roland (Philosophy) Dalhousie Univ.

Pylyshyn, Zenon (Psychology) Univ. West. Ontario

Raab, David H. (Psychology) C.U.N.Y.

Rachlin, Howard (Psychology) S.U.N.Y.

Rajecki, D.W. (Psychology) Univ. No. Iowa

Ramon-Moliner, E. (Anatomy) Univ. Sherbrooke

Ramsay, David J. (Physio.) Univ. Calif.

Rancour-Laferriere, Daniel (Russian) Univ. Calif.

Rao, K.R., Inst. Parapsychology

Rathmayer, Werner (Biology) Univ. Konstanz

Ray, Wm. J. (Psychology) Penn. St. Univ.

Reed, Adam V. (Psychology) New Sch. Social Res

Regener, Eric (Comp. Sci.) Concordia Univ.

Reichert, Thomas A. (Biomed. Eng.) Carnegie-Mellon Univ. Reid, William H. (Psychiatric Inst.) Univ. Neb. Med. Ctr. Reiss, R.F., Lower Heyford

Rey, Georges (Philosophy) S.U.N.Y.

Reynolds, Peter C. (Anthropology) Australian Natl. Univ.

Rice, Charles E., Ed., The Psychological Record, ex. off.

Rife, David C., Clewiston

Ringle, Prof. Martin, Ed., Sistm. Quarterly, ex off.

Ritter, Walter (Neuroscience) Einstein Coll. Med.

Roberts, T.D.M. (Physiology) Univ. Glasgow

Roberts-Gray, Cynthia, Perceptronics Inc.

Robinson, Daniel N. (Psychology) Georgetown Univ

Rocha-Mirando, C.E. (Neurobiology) Univ. Rio de Janeiro

Rodgers, R.J. (Psychology) Univ. Bradford

Roland, Per (Clin. Phys.) Bispebjerg Hosp.

Rolls, Barbara J. (Psychology) Univ. Oxford

Romeo, Luigi, Ed., Ars Semiotica, ex off.

Roodin, Paul (Psychology) S.U.N.Y.

Rorty, Richard (Philosophy) Princeton Univ.

Rose, Steven (Biol.) Open Univ.

Rosenbaum, David A., Bell Laboratories

Rosenblatt, Jay S. (Animal Beh.) Newark Coll.

Rosenthal, David M. (Philosophy) C.U.N.Y.

Rosenthal, Robert (Psychology) Harvard Univ.

Rosenthal, Ted L. (Psychiatry) Univ. of Tenn.

Rosenzweig, Mark R. (Psychology) Univ. Calif., ex off.

Ross, Barbara (Psychology) Univ. of Mass., ex off.

Ross, John R. (Foreign Lit.) Mass. Inst. Tech.

Ross, Robert N. (Psychiatry) Boston Univ. Med. Ctr

Routtenberg, Aryeh (Neurosci.) Northwestern Univ. 
Rowland, N. (Psychology) Univ. Pittsburgh Royce, Joseph R. (Theoretical Psych.) Univ. Alberta Rubens, Alan B. (Aphasia Cen.) Hennepin Cty. Gen. Hosp. Rubin, Donald B. (Data Analy. Res.) Educational Testing Serv. Rumbaugh, Duane M. (Psychology) Georgia St. Univ. Rush, Douglas K. (Primate Lab.) Univ. Wisc. Rutter, Michael (Child Psychiat.) Inst. Psychiatry Ryan, T.A. (Psychology) Cornell Univ. Saayman, G.S. (Psychology) Univ. Cape Town Sacks, Sheldon, Ed., Critical Inquiry, ex. off. Sakitt, Barbara (Psychology) Mass. Inst. Tech. Salzen, Eric A. (Psychology) Univ. Aberdeen Salzinger, Kurt (Appl. Exp. Psy.) Natl. Science Fnd Sankowski, Edward (Philosophy) Ohio St. Univ. Sarles, Harvey (Anthropology) Univ. Minnesota Satoh, Toyohiko (Physiology) Aichi-Gakuin Univ. Savage, C. Wade (Philosophy) Univ. Minnesota Savage-Rumbaugh, E. Sue (Yerkes Primate Ctr.) Emory Univ. Savin, Harris B., (Psychology) Thompson, Penn. Scandura, J.M. (Education) Univ. Penn. Schallert, Timothy (Psychology) Univ. Texas Schank, Roger C. (Comp. Sci.) Yale Univ. Schegloff, Emanuel A. (Sociology) Univ. Calif. Scheibel, Arnold B. (Anatomy) Univ. Calif. Schlag, John (Anatomy) Univ. Calif. Schmidt, Albert L. (Psychology) Southern Illinois Univ. Schmuller, Joseph (Psychology) Clark Univ. Schubert, Glendon, Univ. Hawaii Schusterman, R.J. (Psychology) Calif. St. Univ. Schwartz, Robert (Philosophy) C.U.N.Y Scott, J.P. (Ctr. Res. Soc. Beh.) Bowling Green St. Univ. Searle, Leroy F. (English) Univ. Washington Sebeok, Thomas A. (Language Ctr.) Indiana Univ. Segal, Evalyn F. (Psychology) San Diego St. Univ. Segundo, J.P. (Anatomy) Univ. Calif. Sekuler, Ronald (Psychology) Northwestern Univ. Senault, B., Lab. Le Brun

Severs, Walter B. (Pharmacology) Penn. St. Univ. Shallice, T. (Psychology) Med. Res. Council Shaver, Phillip (Ctr. Hum. Rel.) New York Univ. Shaw, Robert E. (Psychology) Univ. Connecticut Shebilske, Wayne (Psychology) Univ. Virginia Siegel, Jane A. (Psychology) Univ. West. Ontario Siegel, Wm. (Psychology) Univ. West. Ontario Siegel, Michael I. (Anthropology) Univ. Pittsburgh Siegler, Robert S. (Psychology) Carnegie-Mellon Univ. Sigel, Irving E., Educational Testing Serv. Siggins, George R. (Behav. Neurobrol.) Salk Inst. Silverman, Irwin (Psychology) York Univ. Simon, Thomas W. (Philosophy) Univ. Florida Simons, Ronald C. (Psychiatry) Michigan St. Univ. Singer, Jerome, Uniformed Serv. Univ. Hlth. Sci. Slobin, Dan I. (Psychology) Univ. Calif. Sloman, Aaron (Cog. Studies) Univ. Sussex Sluckin, W. (Psychology) Univ. Leicester Smale, Gerald G., Natl. Inst. Soc. Work Smart, J.J.C. (Philosophy) Australian Natl. Univ. Smedslund, Jan (Psychology) Univ. Oslo Smith, Barry H. (Surgical Neurology) N.I.H. Smith, Euclid O. (Yerkes Primate Ctr.) Emory Univ. Smith, N.V. (Linguistics) Univ. College London Snowdon, Charles T. (Psychology) Univ. Wisc. Soltysik, S.S. (Mental Retard. Res. Ctr.) Univ. of Calif. Somit, Albert (Political Sci.) S.U.N.Y.

Somjen, George G. (Physiology) Duke Univ. Med. Ctr. Sontag, K.H. (Pharmacology) Max-Planck-Inst. Sperling, George (Psychology) New York Univ Spinelli, D.N. (Comp. Sci.) Univ. Mass. Springer, Sally P. (Psychology) S.U.N.Y. Staats, A.W. (Psychology) Univ. Hawaii Staddon, J.E.R. (Psychology) Duke Univ. Stanton, Robin B. (Comp. Sci.) Australian Natl. Univ. Steele-Russell, I. (Neural Mechanisms) Med. Res. Council Steffe, Leslie P. (Math. Education) Univ. Georgia Steffens, A.B. (Zoology) Groningen Univ. Stein, Barry E. (Physiology) Med. Col. Virginia Stein, D.G. (Psychology) Clark Univ. Stein, John (Physiology) Oxford Univ.
Stein, Paul S.G. (Biology) Washington Univ. Steklis, Horst D. (Anthropology) Rutgers Univ. Stenlund, Sören (Philosophy) Uppsala Univ. Stephens, J.A. (Physiology) St. Thomas Hosp. Med. Sch. Steriade, Mircea (Physiology) Univ. Laval Sterling, Peter (Anatomy) Univ. Penn. Sternberg, Robert J. (Psychology) Yale Univ. Stewart, Charles G. Jr., New Orleans Stich, S.P. (Philosophy) Univ. Maryland Stoper, Arnold (Psychology) Calif. St. Univ. Stuart, Douglas G. (Physiology) Univ. Arizona Suppes, Patrick (Philosophy) Stanford Univ. Sutherland, N.S. (Psychology) Sussex Univ. Suzuki, Yuko Tsukada (Zoological Inst.) Hokkaido Univ. Szentagothai, J. (Anatomy) Semmelweis Univ. Taylor, David A. (Psychology) Univ. Rochester Teevan, Richard C. (Psychology) S.U.N.Y. Terrace, H.S. (Psychology) Columbia Univ. Terry, W. Scott (Psychology) Univ. No. Carolina Thomas, Arthur J. (Anatomy) Univ. Lausanne Timo-Iaria, Cesar (Neurophysiol.) Univ. São Paulo Tobias, Peter (Anthropology) Rockford Coll. Tombol, Terez (Anatomy) Semmelweis Univ. Townsend, James T. (Psychology) Purdue Univ. Treisman, Michel (Psychology) Univ. Oxford Troost, B. Todd (Neurology) Pittsburgh V.A. Hosp. Tucker, John (Math.) Univ. Waterloo

Tweney, Ryan D. (Psychology) Bowling Green St. Univ. Ullman, S. (Artif. Intell. Lab.) Mass. Inst. Tech.

Ursin, Holger (Psychology) Univ. Bergen Uttal, William R. (Soc. Res.) Univ. Michigan Uzgiris, Ina C. (Psychology) Clark Univ.

Valleala, P. (Physiology) Univ. Turku Valsiner, Jaan (Psychology) Tartu St. Univ. Van Den Berghe, Pierre L. (Sociology) Univ. Washington Vanderweele, Dennis A. (Behav. Neurol.) N.Y.U. Med. Center Vanderwolf, C.H. (Psychology) Univ. Western Ontario Van Valen, L. (Biology) Univ. Chicago Varela, Francisco, Naropa Inst. Vetta, Atam (Math.) Oxford Poly. Villablanca, Jaime (Psychiatry) Univ. Calif. Von Heyden, H. (Psychology) Gottingen Med. Klin. Voneche, Jacques (Psychology) Univ. Geneva Von Foerster, Heinz, Pescadero von Glaserfeld, E. (Psychology) Univ. Georgia Wagman, Althea M.I., Md. Psychiatric Res. Ctr. Waldbillig, Robert J. (Psychology) Univ. Florida Wallace, Meredith (Psychology) La Trobe Univ. Wallach, Hans (Psychology) Swarthmore Coll Walsh, E.G. (Physiology) Univ. Edinburgh Walter, D.O. (Brain Res. Inst.) Univ. Calif. Wandell, B.A. (Psychology) Stanford Univ. Ward, Mark F. (Philosophy) Univ. Minnesota Warren, John M. (Anim. Behav. Lab.) Penn. St. Univ. Warren, Richard M. (Psychology) Univ. Wisc. Wason, Peter (Psycholing.) Univ. Coll. Lond. Wasserman, E.A. (Psychology) Univ. Iowa Wasserman, Gerald S. (Psychology) Purdue Univ. Wassermann, Gerhard D. (Biology) Univ. Newcastle-Upon-Tyne Weeks, J. (Biology) Univ. of Calif.

Weight, Forrest F., N.I.A.A.

Weimer, Walter B. (Psychology) Penn. St. Univ. Weiner, Bernard (Psychology) Univ. Calif.

Weinstein, Sidney, Neurocommunication Res. Labs. Inc., ex off. Weiss, K. (Physiology) Columbia Coll. Phys. Surg. Welch, Robert B. (Psychology) Univ. Kansas Welker, W.I. (Neurophysiology) Univ. of Wisc. Wetherick, N.E. (Psychology) Univ. Aberdeen Whitaker, Harry A. (Psychology) Univ. Rochester White, Sheldon H. (Educ.) Mass. Inst. Tech. Wiepkema, P.R. (Zoology) Univ. Groningen Wiesendanger, M. (Physiology) Univ. Fribourg Wiggins, Richard C. (Neurobiol.) Univ. Texas Wilkins, Robert H. Ed., Neurosurgery Wilkins, Wallace (Psychology) Univ. Maine Wilks, Yorick (Linguistics) Univ. Essex Wilson, David L. (Physiol.) Univ. Miami Wilson, E.O. (Agassiz Museum) Harvard Univ. 
Winn, Wandal W., Anchorage, AK

Winograd, Terry (Artif. Intell. Lab.) Stanford Univ.

Witelson, Sandra F., Chedoke-McMaster Centre

Wohlwill, Joachim F. (Man-Envir, Rel.) Penn. St. Univ.

Wolpe, J. (Psychiatry) Temple Univ. Med. Sch.

Wong, Paul T.P. (Psychology) Univ. Calif.

Wong, Roderick (Psychology) Univ. of Brit. Col

Wood, R.J. (Psychology) Univ. Oxford

Wood, W.S. (Psychology) Drake Univ.

Woody, Charles D. (Neuropsychiat. Inst.) Univ. Calif.

Wright, John W. (Psychology) Washington St. Univ.

Wyler, Allen R. (Neurol. Surgery) Univ. Wash. Sch. Med.

Wyers, Everett J. (Psychology) S.U.N.Y.
Wyrwicka, Wanda (Anatomy) Univ. Calif.

Yeni-Komshian, Grace (Psychology) Univ. Maryland Yonas, Albert (Child Dev.) Univ. Minn.

Young, Andrew (Psychology) Univ. Lancaster

Zacks, James L. (Psychology) Michigan St. Univ.

Zaidel, Eran (Biology) Calif. Inst. Tech.

Zernicki, Boguslaw (Neurophysiol.) Nencki Inst.

Zielinski, Kazimierz (Neurophysiol.) Nencki Inst

Zucker, S.W. (Elect. Engr.) McGill Univ.

Zuckerman, Carl B. (Psychology) C.U.N.Y.

Zuckerman, Marvin (Psychology) Univ. Delaware

Zurif, Edgar (Aphasia Res.) Boston Univ. Med. Cir. 